\title{
THE EFFECTS OF INHALATION OF HELIUM MIXED WITH OXYGEN ON THE MECHANICS OF RESPIRATION ${ }^{1}$
}

\author{
By ALVAN L. BARACH wITH THE TECHNICAL ASSISTANCE OF MORRIS ECKMAN \\ (From the Department of Medicine, College of Physicians and Surgeons, \\ Columbia University, and the Presbyterian Hospital, New York)
}

(Received for publication September 11, 1935)

In previous reports, it has been pointed out that helium is an inert gas with a density one-seventh that of nitrogen and one-eighth that of oxygen, and that a mixture of 80 per cent helium and 20 per cent oxygen has a weight one-third that of a comparable volume of air $(1,2,3)$. The proposal of the use of helium with oxygen as a therapeutic gas was based on the conception that a lighter gas mixture could be moved to and from the lungs more easily than air in those clinical conditions in which difficulty in air movement was present. Since, in normal respiration, only very slight pressures are developed in the tubular system leading to the lungs, the employment of a gas lighter than air. would not be expected to create marked changes in the force required for air movement. However, when obstruction to the flow of air is present, as in asthma and in pathological narrowing of the trachea and larynx, a marked increase in pressure becomes necessary to transport an adequate volume of air through the respiratory passages. The clinical application of a helium-oxygen mixture one-third as dense as air was therefore first made in such cases (4).

The present paper deals with the physiological alterations produced on the mechanics of respiration by the substitution of a helium-oxygen gas mixture for air in those conditions in which increased air pressure in the tubal respiratory system had become necessary to carry on an adequate pulmonary ventilation. Observations on human beings and on dogs are reported in which respiratory obstruction was produced and in which severe dyspnea without obstruction was induced.

\section{METHODS}

In the experiments carried out on dogs, anesthesia was obtained by the intraperitoneal injection of nembutal.

1 The author wishes to acknowledge assistance rendered to him by the U. S. Public Health Service, the Bureau of Medicine and Surgery, U. S. Navy, and the Linde Air Products Company, and to express his appreciation for their aid.
The dose varied from $25 \mathrm{mgm}$. to $40 \mathrm{mgm}$. per kilo body weight. A tracheotomy was performed, and the trachea connected by a short piece of tubing which did not enlarge the dead space further than that present in the natural architecture of the animal. The tube was connected to a Benedict basal metabolism apparatus which was used with a motor blower unit that circulated the gas through soda lime. A slight positive pressure in the system appeared desirable. The tidal air was graphically recorded. The tube that led from the trachea was tapped by hard rubber tubing and connected to a water manometer, the distal end of which was equipped with a delicate float that carried a pen to record the pressure differences that obtained in the trachea. Needles were introduced into each pleural cavity and connected by hard rubber tubing to similar water manometers in order to obtain graphic records of the intrapleural pressure. A small volume of air, 5 to 15 cc., was admitted into the pleural cavity. In addition, in some experiments, a cannula was passed into the abdominal cavity in order to obtain simultaneous records of the change in the abdominal pressures. It was necessary to surround the cannula in the abdomen with a wire mesh screen in order to prevent apposition of the intestines against the cannula. Laparotomy was therefore performed, and the closure was sealed after suture with collodion.

Simultaneous graphic records were thus obtained of the tidal air, pulmonary ventilation, manometric pressure differences in the pleural cavities and in the abdominal cavity. Experiments were conducted with the animal breathing against variable resistances to the flow of different gas mixtures. The depth of anesthesia was found to be of considerable importance in obtaining differences in the response to the inhalation of helium-oxygen mixtures as compared with air. Nembutal is a depressant to the respiratory center, and in deep anesthesia the favorable reaction to the inhalation of a lighter than air gas mixture was obscured. The effect was comparable to that of morphine which reduces the sensitiveness of the respiratory center to oxygen-want as well as to respiratory resistance. Difficulties were also encountered in inadvertent production of artificial pneumothorax which prevented the accurate representation of pleural pressures Furthermore, variations in behavior of the animal to respiratory obstruction at times increased the difficulty of comparing the response to the inhalation of air and to that of a helium-oxygen mixture. This was especially true when long-continued fatigue was present. The experiments were further complicated by the fact that breathing through resistance was followed by pathological 
changes in the lungs which increased progressively as the experiments were continued. When markedly increased negative intrapleural pressures developed, a transudation of blood and serum took place into the alveolar cells, which added the factor of increasing anoxemia.

Experiments were performed on thirty dogs for periods of three to six hours. In five instances, it was possible to obtain relatively constant control periods in which the animal breathed helium-oxygen mixtures. The alterations in these instances were characteristically consistent and reversible. In the other experiments, either failure to obtain complete technical control of the experimental procedures or the variably progressive course of respiratory and circulatory failure interfered with obtaining consistent results.

In the human beings, anesthesia was not employed. Individuals breathed through narrowed orifices for a variable period. Measurements were taken of the tidal volume, the pressure in the extension of the pulmonary airway that led from the subject's mouth, and the external abdominal pressure. The last was obtained by passing around the abdomen a blood pressure cuff, producing against the abdomen a pressure of $15 \mathrm{~mm}$. $\mathrm{Hg}$., and recording the pressure differences graphically by means of the water manometer with pen attached. These abdominal pressures were not intended to represent the absolute internal abdominal pressure but relative differences superimposed on a basic pressure of $15 \mathrm{~mm}$. $\mathrm{Hg}$. Unless this basic pressure is first applied to the abdomen, the looseness of the cuff prevents accurate estimation of relative differences.

\section{OBSERVATIONS}

\section{A. Artificially induced dyspnea and respiratory obstruction in normal individuals}

When an individual breathes slowly and quietly through a tube 2 inches long and 1 inch in diameter, no discernible pressure either during inspiration or expiration is developed in a tubal extension of the pulmonary airway. Thus, if the tube that projects from the patient's mouth is tapped by one-quarter inch tubing and connected distally to a water manometer, quiet breathing does not affect the water level in the two arms of the manometer. A graph obtained on a moving drum which protrays any change in water level shows merely a straight line. However, if severe dyspneic breathing is artificially maintained, well marked oscillations of the water level develop. A normal man who breathed violently through a tube 2 inches in length and 1 inch in diameter, developed a total pressure difference between the levels of the manometer of $8 \mathrm{~cm}$. of water when air was inhaled. When he was placed in a tent inhaling
80 per cent helium and 20 per cent oxygen, the same type of violent dyspnea was accompanied by a total pressure difference of $6 \mathrm{~cm}$. of water. In this case, there was no external obstruction to the flow of gas but the heightened velocity of flow developed frictional resistance in the tubal respiratory system and, therefore, necessitated increased pressures for the transport of air. The lowering of pressure which took place when helium-oxygen mixtures were inhaled is due to the decreased density of this gas.

In order to obtain simultaneous records of the pulmonary ventilation and the pulmonary pressure, a short experiment was performed with the subject breathing into a recording spirometer without valves or soda lime or tubing. In this way resistance was reduced to a minimum. Markedly increased tidal volume and respiratory rate were accompanied by elevated pressures which were greatly lessened when a helium-oxygen mixture was substituted for air (Figure 1). When rapid, shallow breathing was instituted, the pressure changes which developed in the system were too rapid and small to elicit comparisons between air and a helium oxygen mixture. The effect of helium in non-obstructive respiration would appear to be more manifest in types of dyspnea in which increased tidal volumes and increased respiratory rates were both present; however, it has not been possible at the present to determine the effect on respiratory fatigue of a one-third reduction in the total mass of gas used in ventilating the lungs. A dyspneic patient, whatever the character of his respiration, might breathe 45 pounds of air in 24 hours which could be substituted for 15 pounds of helium-oxygen gas at a comparable oxygen tension.

When there is obstruction to the flow of air in any part of the tubular system, there is immediately developed a necessity for an increase in the pressure required for inspiration and expiration; consequently the effect of substitution of a lighter gas mixture is immediately apparent. In an experiment previously reported (2), an individual breathed through an orifice one-eighth inch in diameter into a spirometer' of the basal metabolism type in which valves were used to circulate the air through soda lime. A record was obtained of his tidal air and the pressure produced in the extension of the pulmonary airway, which was tapped 
one inch from the patient's mouth. A comparison between the effect of breathing air (nitrogen 79 per cent, oxygen 21 per cent) and a comparable respirable helium-oxygen mixture (helium 79 per cent, oxygen 21 per cent), showed that the tidal air was approximately the same, being increased in both instances, with a slower respiratory rate. However, the total pressure developed in the tubal system between inspiration and expiration was $10.2 \mathrm{~cm}$. of water when air was breathed and 4.6 $\mathrm{cm}$. when the helium-oxygen mixture was inhaled -a reduction of 54.9 per cent. This decrease in the total range of pressure during the respiratory cycle due to the inhalation of a lighter gas mixture is an indirect estimate of the saving in pulmonary effort obtained in conditions in which there is

NITROGEN 79\%-OXYGEN 21\% HELIUM 79\%-OXYGEN $21 \%$

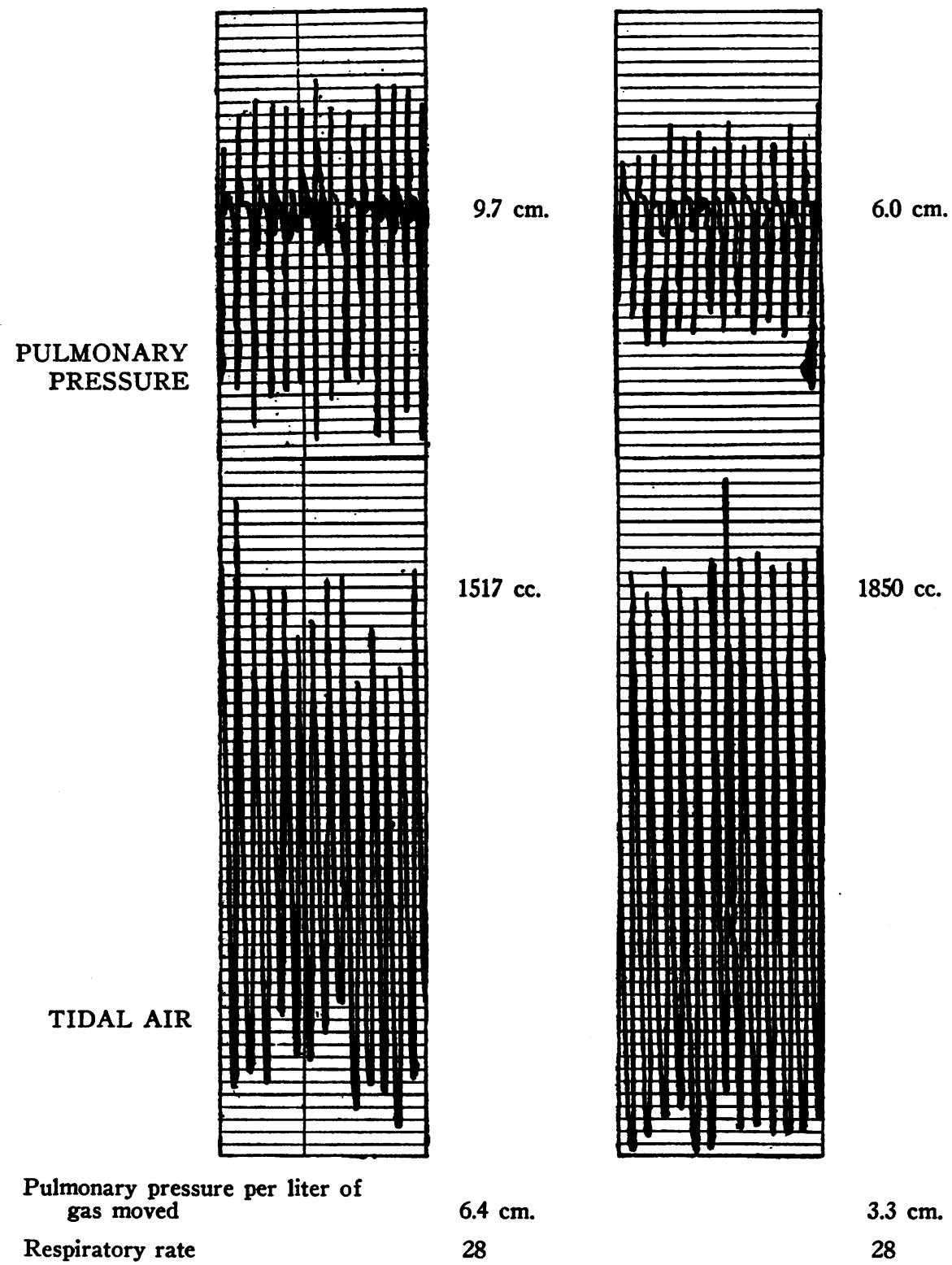

Fig. 1. Reduction in Pulmonary Pressure When Helium-Oxygen Mixture is Inhaled Instead of Air During Violent Dyspnea-Without Resistance. 
obstruction or narrowing in the tubular respiratory system. It may be mentioned that inhalation of pure oxygen is similar to air in its effect on tidal air and pulmonary pressure, oxygen being slightly heavier than air.

The graphic record of the pulmonary pressure showed in the case of air a marked widening at the top of the curve, which indicated that expiration was achieved by the maintenance of a high expiratory pressure, whereas during the inhalation of helium and oxygen there was a prompt fall of the curve at the onset of expiration, representing a decreased need for a continuation of the high expiratory pressure. The clinical significance of this in asthma and obstructive lesions in the larynx and trachea may be visualized as a decreased backward pressure on the alveolar sacs within the lung, and consequently a decreased distension of the alveolar walls, which in turn tends to prevent emphysematous changes in the lung.

A similar experiment was performed on another individual in whom the tidal air and the external abdominal pressure were obtained while he was breathing through an orifice $3 / 32$ nds of an inch in diameter. During the inhalation of air, tive tidal air was markedly increased with a marked slowing of respiratory rate (Figure 2). When the helium-oxygen mixture was inhaled, the tidal air tended to approach normal respiration, the rate being increased and the tidal air lowered. Individuals differ in their response to helium when the respiration is attempted through a narrowed orifice. When air is inhaled, most normal subjects exhibit a slow deep respiration changing over to a more rapid shallow breathing as respiratory fatigue takes place (5). The influence of helium generally manifests itself by lessening the resistance factor, i.e., the breathing is not so deep nor as slow; in a few cases, the tidal volume remains the same. In both types of response the pressure in the pulmonary tubal system is decreased 30 to 50 per cent. The above graph (Figure 2 ) reveals that the external abdominal pressure was decreased approximately 50 per cent during the inhalation of the helium-oxygen mixture as compared to air. Furthermore, the curve of the abdominal pressure is different in quality, being sharper at the onset of inspiration and expiration when a helium-oxygen mixture is breathed, and widened during the breathing of air. (The ascending stroke of the pressure curve represents inspiration and the descending stroke expiration.) Obviously, greater effort is necessary both for inspiration and expiration when air is inhaled. During the inhalation of helium-oxygen mixtures, there is a diminished sensation of air-hunger. Furthermore, when 85 per cent helium is breathed with 15 per cent oxygen, there is more striking alleviation of dyspnea, even though a mild anoxemia is produced. This applies to the early period of resistance experiments. The primary factor in the dyspnea of obstructive respiration appears, therefore, to be a physical interference with the flow of air in and out of the lungs at a velocity and pressure to which the individual is accustomed, rather than oxygen-want or carbon dioxide excess. Undoubtedly, the fundamental requirement of the tissues for oxygen is the basic chemical stimulus for increased pulmonary effort, but at the onset of obstructive respiration there is no oxygen-want nor carbon dioxide retention. The cause of the sensation of dyspnea must be sought for in the disturbance of the local pulmonary vagal nerve endings which are stimulated by an imposed hindrance to the accustomed rate of filling and emptying of the lung. This belief is borne out by the actual response of patients with asthma and those who have obstruction in the larynx and trachea, a report of which is made in another communication (4), as well as by data now to be presented.

Numerous experiments were performed on normal individuals who were exercising during the inhalation of helium-oxygen mixtures and air. Measurements of the tidal air, total pulmonary ventilation, and the pulmonary pressures were recorded by connecting the patient by a mouthpiece to a basal metabolism apparatus. In all these experiments, there was the added factor of resistance of the apparatus itself. In every case, there was a decrease in the pressure in the pulmonary airway of between 25 and 50 per cent. Similar results were obtained by mild exercise in compensated cardiac patients. It does not appear necessary to report these in detail since the fundamental principle has been expressed in previous graphs, namely, that where there is any resistance to the flow of air the inhalation of a helium-oxygen 
छ்

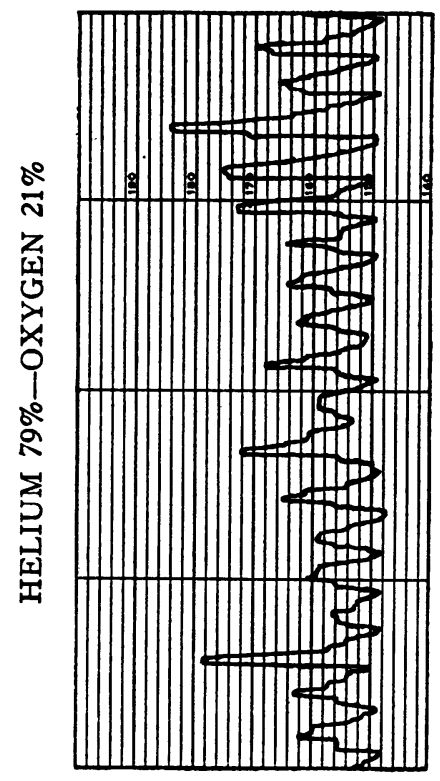

$\dot{g}$
$\stackrel{ }{ }$

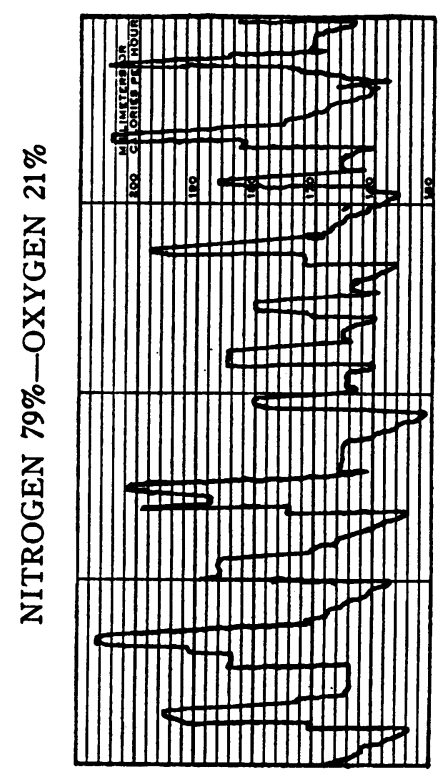

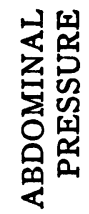

ن்

宅

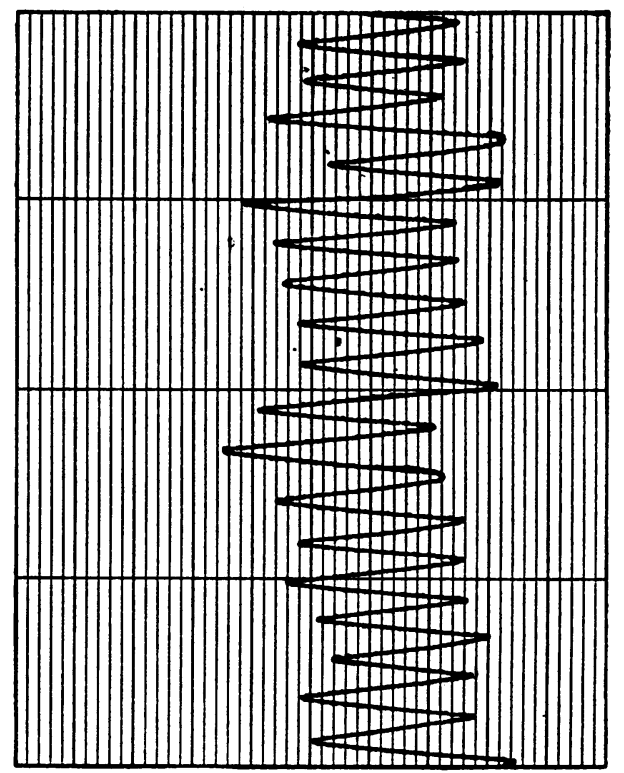

ฮู

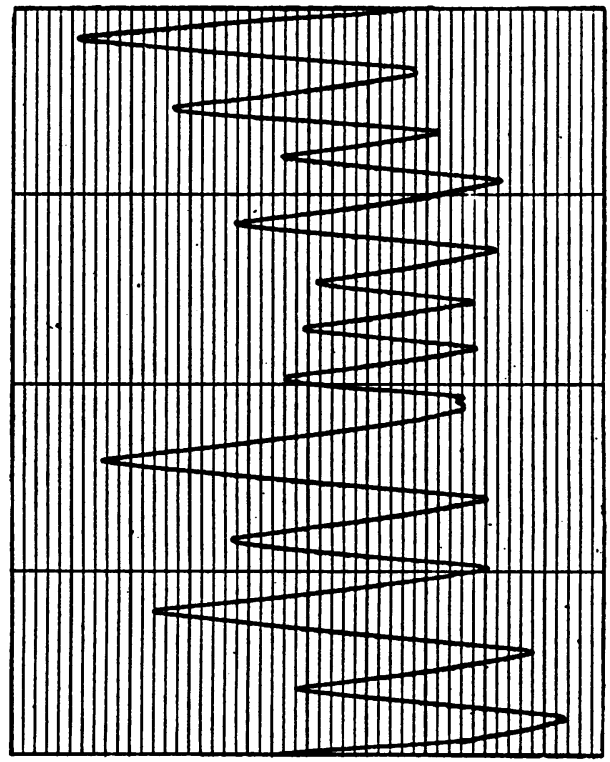

吕

选
:

영

변은

范

要

突

5

렬

西

营

政

秋

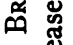

政

- $\quad 5$

造览

尝总

岁䒺

1

嵌

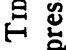

究

啊

용

员

昱.

。

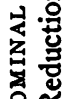

ํㅛㅇ

<

சुำ ن

옹

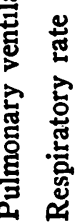


mixture can proceed with lessened pressure within the tubular system. The effect of the inhalation of the lighter mixture on the tidal air varies with the individual and with the type of dyspnea existing. In dyspnea with hyperventilation, produced by obstruction to the flow of air, the influence of helium is generally to decrease the tidal air and the total pulmonary ventilation. In dyspnea produced by exercise in normal men, the tidal air and the total pulmonary ventilation may be the same or slightly increased. However, it is impossible to measure the tidal air without some degree of resistance in the measuring apparatus, so that one cannot rule out entirely the factor of resistance to the flow of air, when recording pulmonary ventilation, even though no obstruction is introduced into the airway itself.

In summary then, the substitution of heliumoxygen mixtures for air is followed by a decrease in the pressures developed during the respiratory cycle in man, in all instances in which there is increased resistance to the movement of respired air. The tidal air and the total pulmonary ventilation are generally decreased, accompanied by a sensation of diminished air hunger. In normal individuals breathing without obstruction, severe dyspnea, induced either by artificial voluntary increase in pulmonary ventilation or by exercise, takes place with a much smaller increase in the pressure in the pulmonary airway when heliumoxygen mixtures are inhaled instead of air. The tidal air under these circumstances cannot be recorded since the measuring apparatus itself would introduce some resistance. However, in the presence of this resistance, the tidal air is either the same or is increased.

\section{B. Respiratory obstruction in tracheotomized dogs}

When a dog's trachea is connected to the recording spirometer of a basal metabolism apparatus, the resistance of the apparatus is at once introduced. This resistance may be lessened by a motor blower unit, but cannot be entirely removed. The pressure existing in the pulmonary airway represents the pressure against which the animal is breathing. In the presence of this slight resistance, the pressure in the pulmonary airway is decreased 20 to 30 per cent when helium-oxygen mixtures are inhaled as opposed to air, a result comparable to that observed in human beings previously reported (2). The intrapleural and abdominal pressures are, however, not measurably altered in quiet respiration in the absence of resistance to breathing. When, however, the tube connected to the trachea is constricted by the insertion of a cork with an orifice of $3 / 32$ nds of an inch, marked changes take place. It must be borne in mind that the pressure in the pulmonary airway is obtained by tapping the tube connecting the trachea at a point between the trachea and the resistance, since only in this way is the pressure in the pulmonary airway, in this instance actually the intratracheal pressure, arrived at. After the resistance has been put in place, the rate of breathing of the dog increases with a simultaneous increase in the inspiratory and expiratory intrapleural and intra-abdominal pressures. This is accomplished by a parallel increase in the intratracheal pressure. For a variable period of onehalf hour to two hours, there are progressive changes generally characterized by development of more rapid and shallow respiration and by increases in all pressure measurements, with irregular periods interposed in which for a time the pressure may be held constant in the pleural cavity, the abdominal cavity and in the trachea. It is during these periods, in which the pressures developed during the respiratory cycle appear to be relatively constant, that comparison of the effect of the inhalation of air and a comparable heliumoxygen mixture may be recorded.

Progressive increase in intrapleural pressure during respiration against resistance. Figure 3 shows the increase in the negative intrapleural pressure range as resistance is introduced in the trachea, namely from $2.2 \mathrm{~cm}$. of water to $5.4 \mathrm{~cm}$. An increased suction pressure is immediately obtained to maintain the accustomed pulmonary ventilation. In this instance, the diameter of the tube connected to the dog's trachea for the 3 hour period of the experiment was $1 / 8$ inch. During the early stages of the experiment, only slight lessening of the intrapleural pressure was accomplished by a change from air to a helium-oxygen mixture. Without any resistance to inspiration, or very slight resistance, no differences whatsoever can be recorded. As the animal continues to 
TME

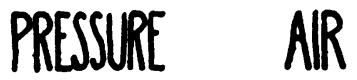

TIME

PRESSPRE

HELLU182\%

PMNEE

RANGE OXYGEN $18^{\circ}$

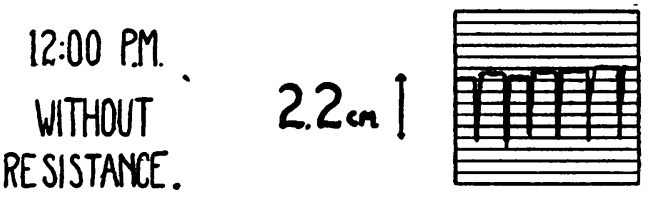

12:15 P.M.
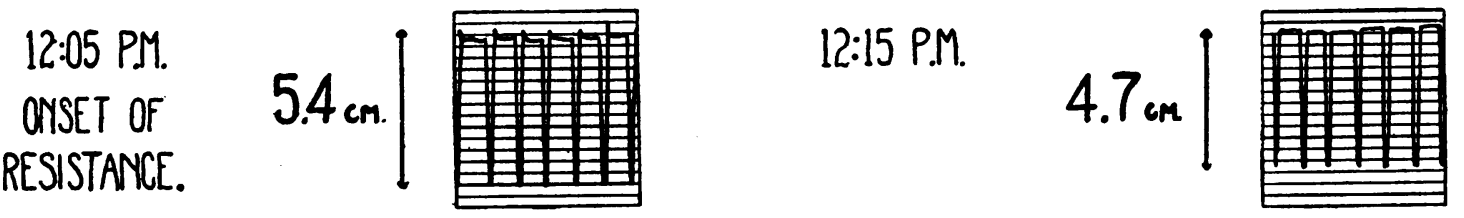

3:30PM

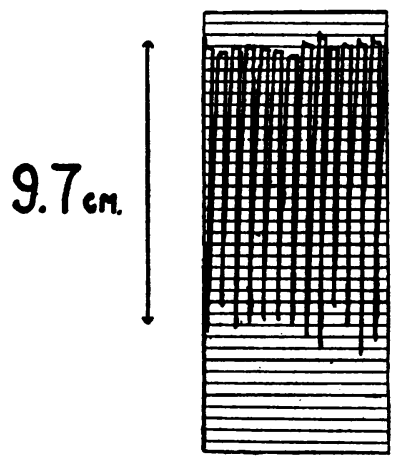

Fig. 3. Progressive Increase in Intrapleural Pressure with Animal Breathing Continuously through $1 / 8$ INCh Orifice

breathe against marked resistance, the intrapleural pressure increases markedly, as seen in Figure 3. The decrease in the total range of pressure which then takes place when helium-oxygen mixtures are inhaled is considerable, in this experiment from $13.0 \mathrm{~cm}$. water to $9.7 \mathrm{~cm}$. water. There were no significant changes in the tidal volume when helium-oxygen mixtures were breathed instead of air. The intratracheal pressure was not recorded. The significance of the progressive increase in intrapleural pressure (chiefly heightened negative pressure) will be discussed later.

Decrease in intrapleural and intrapulmonary pressures with increase in tidal volume when helium-oxygen mixtures are substituted for air. The term "intrapulmonary pressure" refers to the pressure in the tubal extension of the animal's trachea. This measurement is constantly lowered when helium-oxygen mixtures are substituted for air. In Figure 4, observations are recorded on the intrapleural pressure, the intrapulmonary pressure and the tidal volume of a dog three hours after respiration through an orifice $1 / 8$ inch in diameter. At this time, the total pressure between inspiration and expiration in the right pleural cavity was $7.98 \mathrm{~cm}$. when breathing air 


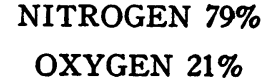

INTRAPLEURAL PRESSURE

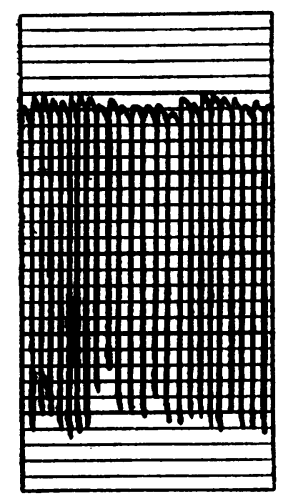

INTRAPULMONARY PRESSURE

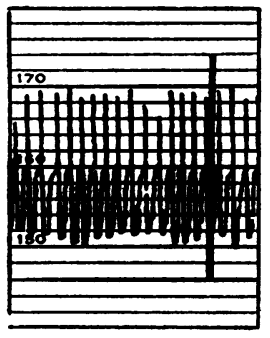

$7.98 \mathrm{~cm}$.

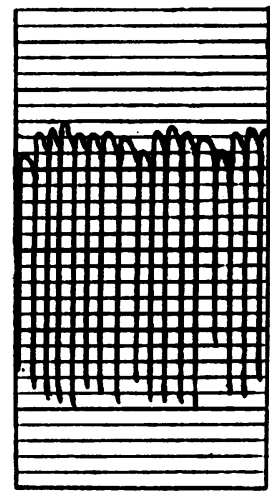

$3.56 \mathrm{~cm}$.

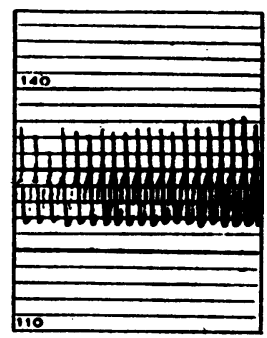

$66 \mathrm{cc}$.

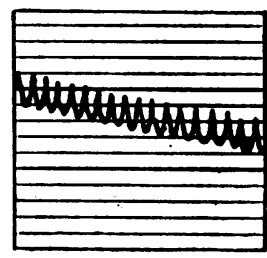

20

$1320 \mathrm{cc}$.
$6.48 \mathrm{~cm}$.

HELIUM 79\%

OXYGEN 21\%

$2.4 \mathrm{~cm}$.

93 cc.

17.75

$1650 \mathrm{cc}$.
Pulmonary ventilation

Fig. 4. Decrease in Intrapleural and Intrapulmonary Pressure, with Increase in Tidal Volume, When Inhalation of Helium-Oxygen Mixture is Substituted FOR AIR

Dog breathed through $3 / 16$ inch and $1 / 8$ inch orifice.

and $6.48 \mathrm{~cm}$. when breathing the mixture of 21 per cent oxygen and 79 per cent helium. ${ }^{2}$ The

\footnotetext{
2 The second decimal points were obtained through mathematical averages of the pressure and not through accuracy of actual measurement. It should also be observed that in the graph which represents the intrapleural pressure during the breathing of air the respiratory rate is increased above that registered by the intrapulmonary pressure and the tidal volume, indicating that they were not taken synchronously in this instance. The rate of respiration was slower when the helium-oxygen mixture was breathed in the portion of the graph taken for repro-
}

pressure in the pulmonary airway extension was $3.56 \mathrm{~cm}$. of water during the inhalation of air and $2.4 \mathrm{~cm}$. during inhalation of the heliumoxygen mixture. This relative reduction obtained throughout the experiment.

The tidal volume throughout the greater part duction; this is not characteristic, as in other instances the breathing seems to increase in frequency when the helium-oxygen mixture is substituted for air. There are uncontrollable periodic variations in the quality of ventilation which are independent of the type of gas inhaled. 
of the experiment remained the same during the breathing of the two mixtures. At the end of three hours, when the respiratory musculature of the animal was fatigued, the tidal volume during the inhalation of air was $66 \mathrm{cc}$. and $93 \mathrm{cc}$. during the inhalation of the helium-oxygen mixture. An increased ventilation was accomplished at a lower intrapleural pressure difference. In the spirometer experiment previously reported $(2,3)$, mechanical lifting of the spirometer bell showed a greater tidal air at a decreased pressure within the spirometer when the helium-oxygen mixtures were used in place of air. This reaction could only be induced at certain times in numerous experiments on the dog, more frequently when extreme fatigue was induced, as by breathing through resistance for three to six hours.

A similar response is shown in Figure 5, in which the helium-oxygen mixture is contrasted to air and 100 per cent oxygen. The dog had been breathing through a $3 / 16$ inch orifice for three hours.

As indicated in the graph, respiration of the helium-oxygen mixture for a short period, even though it had in this instance a lower oxygen concentration, was accomplished with a smaller intrapleural pressure difference than that found during the breathing of air or pure oxygen. At the same time, there was a distinct increase in the tidal air volume when the helium-oxygen mixture was breathed as compared to either air or oxygen. Following the initial reduction in intrapleural pressure when 15 per cent oxygen and 85 per cent helium was inhaled, there gradually developed an increase in respiratory rate and an elevation in intrapleural pressure, due to the development of anoxemia.

This experiment was unusual in that the inhalation of pure oxygen did not immediately slow the respiratory rate. In most of the dog experiments which were carried to the point of severe fatigue, the inhalation of pure oxygen promptly slowed the respiratory rate, and in this way decreased the intrapleural pressure. The decrease in the intrapleural pressure due to oxygen inhalation was directly dependent upon the decrease in rate, for in those instances in which no slowing of the respiratory rate occurred when oxygen was breathed, there was no decrease in intrapleural pressure.

During the early period of an experiment in which a dog breathed through a narrow orifice, for one-half to one hour, depending on the degree of resistance imposed, the substitution of 100 per cent oxygen for air produced no change in the intrapleural pressure. However, during the later stages of the experiment, when respiratory and circulatory failure were impending, the inhalation of increased oxygen concentrations was regularly followed by slowing of the respiratory (and cardiac) rates with a corresponding reduction in intrapleural pressure. It was evident that a severe degree of anoxemia had been superimposed on the previous purely obstructive respiration. There were then two indications for treatment, one, that of a physically light gas to pass the obstruction in the trachea, and two, an increased oxygen concentration to overcome the progressive oxygenwant. Under these circumstances, a comparison was made between the effects of inhalation of increased oxygen concentrations in air with similar oxygen concentrations in helium. The influence of helium could be regularly observed in a lowering of the intrapleural pressure. Increasing the oxygen concentration almost always slowed both the cardiac and respiratory rates, and thus the intrapleural pressure, when comparison was made with air. Accurate contrasts of the difference between nitrogen and helium as the diluent of oxygen were difficult to record in the swiftly altering pathological picture, but it appeared that 30 to 35 per cent oxygen in helium was of greater benefit than any other combination, as portrayed by decreased intrapleural pressure and intratracheal pressure, a slower cardiac and respiratory rate and a larger minute volume. When, for example, an animal was suddenly shifted at the end of an experiment of four to six hours from the inhalation of 30 per cent oxygen and 70 per cent helium to 21 per cent oxygen and 79 per cent nitrogen, an immediate change for the worse took place, manifested by marked increase in intrapleural pressures and in cardiac respiratory rates.

Increasing the oxygen concentration above 35 per cent lost some of the advantages of helium as a physically light element for transport of gas 

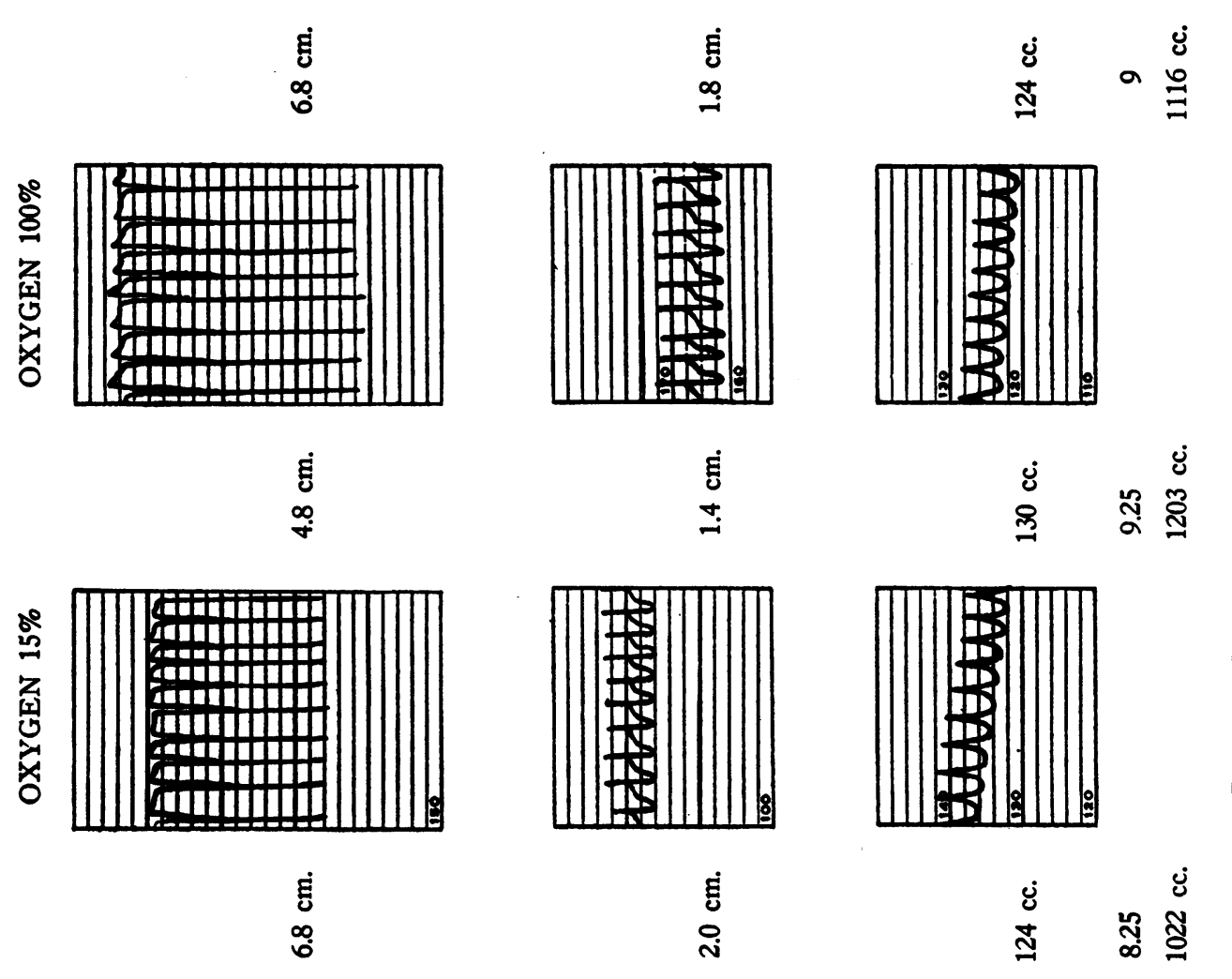

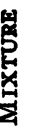
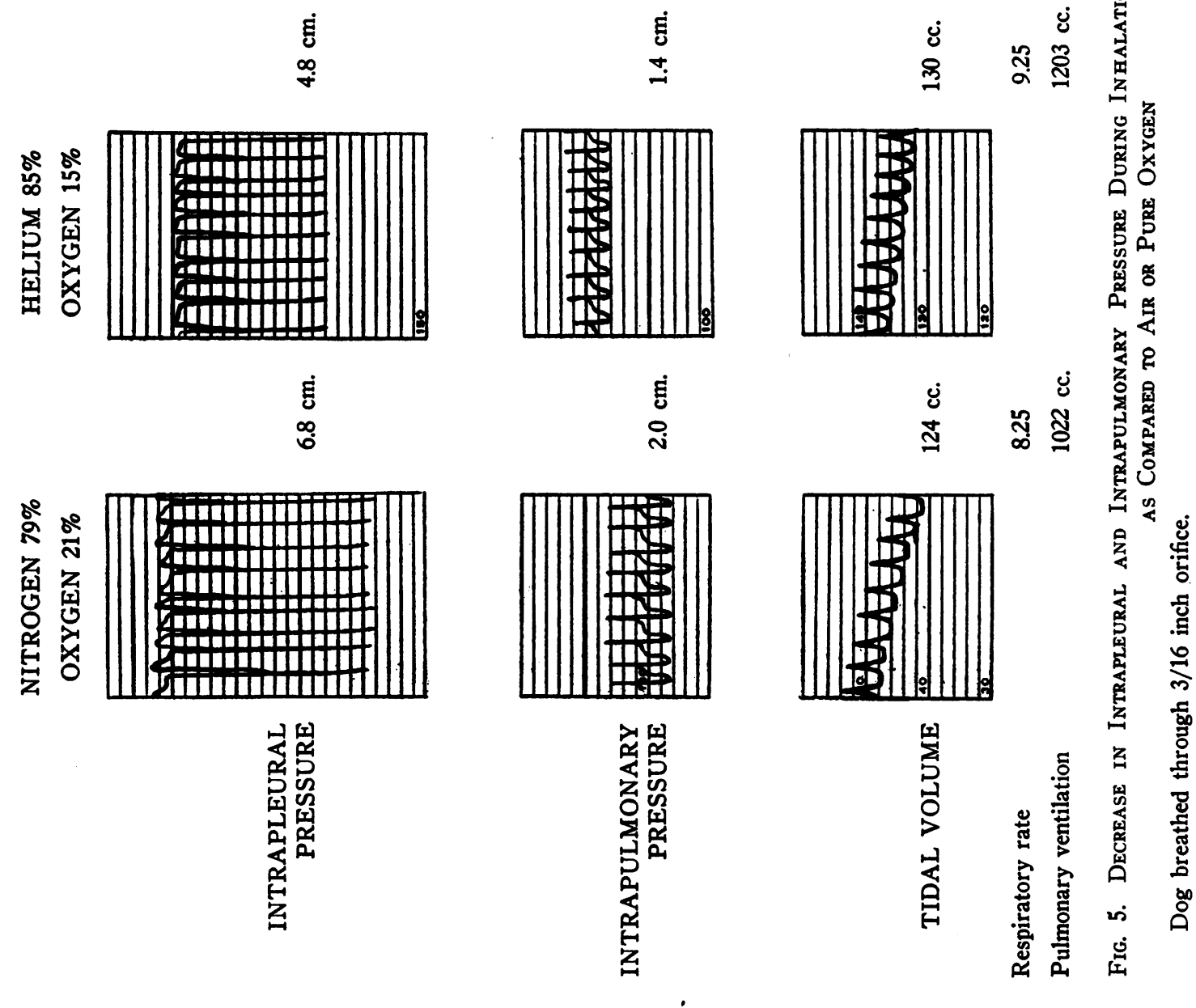
in and out of the chest, whereas lowering the oxygen concentration below 30 per cent added the menace of increased anoxemia. ${ }^{3}$

\section{DISCUSSION}

The clinical situation may be better reviewed in the light of the pathological changes found at autopsy. When dogs were subjected to breathing through narrow orifices for three to six hours, their lungs were found to be grossly congested. Microscopically, vast areas of hemorrhage into the alveolar cells were observed. The exudation of red blood cells and edema fluid as a result of prolonged breathing against resistance has been commented upon by others. In the original studies of Davies, Haldane and Priestly (5), on the manner in which breathing adapts itself to increased resistance, they showed that the normal response in man was slow, deep breathing followed by rapid shallow breathing. The immediate response involved the Hering-Breuer reflex; later anoxemia was added to the picture and hastened the onset of fatigue of the respiratory center. The explanation of the development in animal experiments of pulmonary congestion and edema after continued breathing against resistance was ascribed by Graham (6) to the heightened negative pressures within the chest inducing a seepage of fluid and red corpuscles into the pulmonary parenchyma, a kind of "dry cupping" of the lung (Hoover (7)). Moore and Binger (8) found that these pathological changes occurred in inspiratory obstruction and were lacking in animals subjected to expiratory obstruction.

The heightened negative pressure within the chest would also appear to facilitate the inlet of

3 There is another complicating element in the response of the animal to respiration against resistance, namely, the pressure in the ventilating system. If inspiratory and expiratory flutter valves are used alone, the resistance in the closed system adds an increased burden, whereas increasing the positive pressure by a leak-tight motorblower unit facilitates respiration. (If the blower is not leak-tight, the helium loss will obscure result of experiment.) In further studies in progress, administration of a helium-oxygen mixture under 4 to $10 \mathrm{~cm}$. positive pressure has been found to lower intrapleural negative pressure to a corresponding degree, thus further lessening the consequences of long-sustained heightened negative pressures within the chest, as will be brought out in the forthcoming discussion. blood into the right ventricle and retard the flow into the left. This would result in an accumulation of blood in the lungs with an increased capillary blood pressure, which would seem to be an important factor in the production of intraalveolar exudate and hemorrhage. Welch (9), many years ago, suggested as a cause of edema of the lungs a disproportion in the output of the two ventricles. Recently, investigation (10) has tended to confirm his suggestion, and to indicate that any method which would diminish the negative intrapleural pressure would prevent edema from developing. ${ }^{4}$ Our own findings with the inhalation of helium-oxygen mixtures revealed that the dyspnea of respiratory obstruction, both clinically in asthma and in experimental tracheal constriction in dogs, was more markedly relieved when the gas was administered under positive pressure. During inspiration the gas is partly forced into the lungs, necessitating lower negative pressures within the chest, lessening (theoretically) the disproportion between the output of the two ventricles, and consequently, decreasing the accumulation of blood and serum in the lungs. Since expiratory obstruction alone does not result in intra-alveolar hemorrhage or edema, the slight increase in resistance to expiration is of less pathologic significance in this regard. Further-

4 The evidence referred to above is adequate to prove that edema of the lungs, produced in a rabbit by injection of a large dose of adrenalin, is prevented or ameliorated by breathing air at a pressure greater than the atmosphere. (Johnson (10), Loeb (10).) Johnson observed decreased filling of the left heart and more vigorous contractions of the right chamber when the animal had pulmonary edema. That the ventricles of the heart deliver unequal amounts of blood under these conditions was also noticed by Auer and Gates (10). The formation of edema in the lungs was prevented by artificial respiration, an observation that was first made in experiments of Haven Emerson (10). In all these studies, it was clear that increased negative intrapleural pressures were present; it seems most likely that a cupping action on the alveoli and an unequal output of the two chambers of the heart were secondary to reduction of intra-chest negative pressure. All measures which diminish negative pressure during inspiration would therefore decrease pulmonary edema. Poulton (10) has tried out a suggestion of Plesch to treat pulmonary edema by administering air under positive pressure, and reports relief of dyspnea in some cases. In our clinical studies we have used positive pressures of 4 to $10 \mathrm{~cm}$. in inspiration and expiration in the treatment of both asthma and pulmonary edema. 
more, in asthma, the increased pressure within the tubal respiratory system may be of help in preventing collapse of the small bronchioles when the pressure within the chest becomes higher than that of the atmosphere at the height of expiration, a supposition that seems to be borne out by the response of the patient with asthma to the inhalation of helium-oxygen mixtures under slightly positive pressures.

The sequence of events following inspiratory obstruction appears, therefore, to be as follows: increased negative intrapleural pressures, a disproportion between the output of the two ventricles resulting in an increased capillary blood pressure, accumulation of blood in the lungs, intra-alveolar hemorrhage and edema, progressive anoxemia, finally ending in respiratory and cardiac failure. The usefulness of increased concentrations of oxygen in these conditions is obvious. However, even 100 per cent oxygen cannot prevent ultimate collapse and death, since the difficulty of oxygen (which has approximately the same density as air) entering the lungs perpetuates the need for heightened negative chest pressures. The addition of helium to oxygen tends to aid the primary difficulty of breathing through a narrowed orifice because of the decreased force necessary to transport a light gas. The optimal proportion of helium and oxygen employed in a patient with respiratory obstruction would have to be decided by recognizing the extent to which the factors above discussed were operative in the individual case. The precise pressure at which the gas is administered is an additional factor of definite importance in which further experience is necessary.

In a clinical communication on the therapeutic influence of helium (4), it was found that one infant with laryngeal obstruction did best with a mixture of 30 per cent oxygen and 70 per cent helium, whereas another with tracheal obstruction was more relieved by an oxygen concentration of 24 per cent in the mixture. Elevating the oxygen concentrations substantially above these percentages caused increased dyspnea, whereas lowering them further resulted in cyanosis, increased pulse and respiratory rate. However, patients with chronic continuous asthma were frequently more relieved of dyspnea with a mixture of 17 to 19 per cent oxygen and 81 to 83 per cent helium, than with concentrations of oxygen above these percentages. Furthermore, the slight intensification of anoxemia in the patients with asthma was without the ill effects observed when cases of laryngeal or tracheal obstruction were similarly treated.

An explanation of these differences is presumably present in the experimental facts just recited. In asthma, expiration is more prolonged and difficult than inspiration, and there is less likelihood, in the average case, of extremely high negative pressures in the pleural cavity. However, in obstructive lesions in the larynx or trachea, a high negative intrapleural pressure must be induced to commence the movement of air through the trachea; with severe obstruction the inspiratory stridor is accompanied by a sucking in of the abdomen or chest wall, an occurrence more often seen in infants. In the presence of such marked inspiratory obstruction, the secondary development of edema fluid and red corpuscles passing into the alveolar sacs contributes the dangerous factor of severe anoxemia with its resultant damaging effect on the circulatory and respiratory systems. The indication for increased oxygen concentrations in the atmosphere inhaled is obvious; the use of helium as a diluent facilitates the entrance of the combined gas and tends to prevent fatigue and paralysis of the respiratory musculature.

Since patients with asthma have been treated with helium in the presence of oxygen concentrations lower than air with relief of dyspnea and a clearing up of the various manifestations of fatigue of the respiratory center, it can be safely assumed that marked passive pulmonary congestion, excessively high negative intrapleural pressures and severe anoxemia were absent. Nevertheless, in patients with emphysema and asthma in whom preexisting anoxemia may be severe, the inhalation of helium mixtures with lowered oxygen concentrations should be practised cautiously. The greater the concentration of helium, the more effective will the mixture be in overcoming fatigue of the respiratory musculature and the allied sensation of dyspnea, provided the untoward consequences of anoxemia are avoided.

Since the intrapleural pressures are generally in a negative range, the inhalation of helium- 
oxygen mixtures reduces the negative pressure within the chest. This effect is increased if the gas is administered under positive pressure. At the end of a long experiment on an animal breathing against resistance, there is not only a heightened negative pressure during inspiration but a positive pressure of 1 to $3 \mathrm{~cm}$. of water during expiration. The accumulation of blood and edema in the lungs is probably the most important factor in the development of increased negative and positive pressures required for ventilating the lungs. An additional supposition is that the muscles of respiration do not work synchronously, for which hypothesis further data are necessary. During the end of an experiment, very high intrapleural pressures are at times observed with extremely small tidal volumes. In part, encroachment of congested blood vessels on alveolar spaces and emphysema in other parts, suggests itself as an explanation, but there is a possibility that the respiratory musculature does not contract uniformly under these circumstances.

There remains to be discussed the more general bearing of these results on the concept of airhunger. In presentations of the subject of dyspnea, the immediate cause of the sensation of dyspnea has not always been clearly separated from the cause of the dyspneic state; i.e., the pathological physiology responsible for the sensation of dyspnea. Emphasis has recently been put on the proprioceptive reflexes emanating from the lungs and the chest wall as the cause of cardiac dyspnea, minimizing the chemical factors ordinarily assumed to be operative (Harrison (11)). It can be readily granted that the sensation of airhunger is aroused intracerebrally from nerve impulses traversing the Hering-Breuer reflex arcs and related nerve pathways, and not from a nerve impulse generated by the hemoglobin of the red cell being inadequately equipped with oxygen or unduly burdened with carbon dioxide. However, an insistence on the subjective aspect of the concept of dyspnea limits our understanding of what is happening to the pathological physiology of the respiration and circulation which has evolved the dyspneic state. For when the cause of dyspnea is approached from this point of view, it immediately becomes apparent that an inefficiently contracting heart muscle has produced a train of consequences that interfere with an effortless provision of oxygen to the tissues. The passive pulmonary congestion, the slowed blood flow, the decrease in the oxygen saturation of the arterial blood or the presence of an oxygen debt-all are evidences of deficient ability to supply oxygen to and remove carbon dioxide from the body. The increased pulmonary ventilation, which is a uniform characteristic of cardiac dyspnea, must be viewed, at least in part, as an effort to remedy the failing circulation. Since the inhalation of oxygen generally causes a progressive fall in pulmonary ventilation in patients with congestive failure, as well as improved heart action, both the dyspneic state and the sensation of dyspnea are relieved. There is, therefore, a very real relation between chemical factors and the cause of dyspnea (12). It would be permissible to say only that the cause of the sensation of dyspnea, as a subjective symptom and not an objective pathologic process, is due to disturbed proprioceptive pulmonary reflexes. It must be remembered that all dog experiments cited as bearing on dyspnea are obviously not concerned with dyspnea as a subjective awareness of distressful breathing but with the pathological process which we have been considering.

In obstructive respiration, however, the proprioceptive reflexes have a more profound physiological significance. In three infants who suffered from laryngotracheal obstruction, the relief of dyspnea was due mainly to the decreased effort needed to accomplish an accustomed rate of filling and emptying the lung; the relief of air-hunger was plainly dependent on a more normal stimulation of the proprioceptive reflexes emanating from the lungs and chest wall. However, there was also in these cases, and in the dogs in whom tracheal obstruction was produced, a congestion of the lungs with edema and red corpuscles, which interfered with the normal diffusion of oxygen through the pulmonary membrane. An increased supply of oxygen was also a beneficial factor in the relief of dyspnea; the cause of the dyspneic state was thus in part the development of anoxemia. In asthma, the interference with a normal velocity of pulmonary ventilation and the burdensome effort imposed on the respiratory musculature are responsible not only for the sensation of dyspnea but to a large degree for the 
objective manifestations of dyspnea. In severe cases, as has been mentioned, there are added secondarily the chemical factors of anoxemia and threatened disturbance in acid-base equilibrium. The pathological physiology of air-hunger in obstructive respiration may be compared to that in heart failure. In the latter, the objective physical sign, dyspnea, is caused by an insufficiency of the circulation in its function of maintaining an adequate endowment of the tissues with oxygen and an adequate elimination of carbon dioxide. Only when it is understood as a psychic state of airhunger is it possible to define cardiac dyspnea as due simply to disturbances in reflex nerve pathways from the lungs. In the dyspnea of respiratory obstruction, the disturbance in the proprioceptive reflexes is again the cause of the sensation of dyspnea, and probably initiates increased pulmonary ventilation, but the consequences of obstruction to respiration, such as increased negative pressures within the chest and pulmonary congestion, are fundamental causes of some of the manifestations of obstructive dyspnea.

\section{SUM MARY}

Experiments were conducted on normal human subjects and on dogs to determine the effect of helium mixed with oxygen on certain types of dyspnea. When normal individuals breathe through a constricted orifice, such as $3 / 32$ nds of an inch, respiration slows in rate and increases in tidal volume. The air pressure in the trachea or in a tubal extension of the pulmonary airway is markedly increased. If 100 per cent oxygen is substituted for air, no distinct changes are observed either in the physical measurements or in the sensation of air-hunger. When, however, a mixture of 80 per cent helium and 20 per cent oxygen is inhaled, the pressure in the pulmonary airway is lowered approximately 50 per cent, the respiratory rate is increased toward the normal, and the tidal volume is generally decreased. The sensation of dyspnea is partially relieved.

When normal subjects are made to breathe violently, with markedly increased tidal volumes, increased pressures are present in the pulmonary airway, which are reduced between 30 and 50 per cent by the inhalation of 20 per cent oxygen and 80 per cent helium.
In dogs subjected to breathing through narrowed orifices for three to six hours, the influence of helium-oxygen mixtures was compared to that of air and of 100 per cent oxygen. The inhalation of 20 per cent oxygen and 80 per cent helium was followed by significant decreases in the intrapleural, intra-abdominal and intratracheal pressures. During the later stages of the experiment, the dogs developed marked congestion and edema of the lungs incident to the maintenance of high negative intrapleural pressures. The inhalation of helium 80 per cent and oxygen 20 per cent in some instances resulted in both a reduction in intrapleural pressure and an increase in tidal volume. The inhalation of 100 per cent oxygen under these circumstances improved the condition of the animal by slowing the respiratory and cardiac rates. A mixture of 30 to 35 per cent oxygen with 65 to 70 per cent helium seemed to be of greater benefit than other combinations. In summary, helium decreased the effort of the respiratory musculature and, as a physical function, lowered intrapleural pressure. Oxygen administered in increased concentrations decreased the pulmonary ventilation, and, as a chemical factor, lessened the work of respiration. The presence of nitrogen was detrimental in that it was useful neither physically nor chemically.

The application of these findings to clinical conditions, chiefly asthma and obstructive lesions in the trachea and larynx, as well as their more general bearing on the concept of dyspnea, was discussed.

\section{BIBLIOGRAPHY}

1. Barach, A. L., Rare gases not essential to life. Science, 1934, 80, 593.

2. Barach, A. L., Use of helium as a new therapeutic gas. Proc. Soc. Exper. Biol and Med., 1934, 32, 462.

3. Barach, A. L., Therapeutic use of helium. Anesth. and Analg., 1935, 14, 210.

4. Barach, A. L., Therapeutic use of helium in asthma and obstructive lesions in larynx and trachea. Ann. Int. Med. (To be published December, 1935.)

5. Davies, H. W., Haldane, J. S., and Priestley, J. G., The response to respiratory resistance. J. Physiol., $1919,53,60$.

6. Graham, E. A., Influence of respiratory movements on the formation of pleural exudates. J. A. M. A., 1921, 76, 784. 
7. Hoover, C. F., Respiratory symptomatology. Nelson's Loose Leaf System of Medicine, 1922, 3, 385.

8. Moore, R. L., and Binger, C. A. L., (a) Observations on resistance to the flow of blood to and from the lungs. J. Exper. Med., 1927, 45, 655.

(b) The response to respiratory resistance; a comparison of the effects produced by partial obstruction in the inspiratory and expiratory phases of respiration. J. Exper. Med., 1927, 45, 1065.

9. Welch, W. H., Zur Pathologie des Lungenödems, Virchow's Arch. f. path. Anat., 1878, 72, 375.

10. Meltzer, S. J., Edema; a consideration of the physiologic and pathologic factors concerned in its formation. Am. Med., 1904, 8, 191.

Emerson, Haven, Artificial respiration in the treatment of edema of the lungs. Arch. Int. Med., 1909, 3, 368.

Auer, J., and Gates, F. L., Experiments on the causation and amelioration of adrenalin pulmonary edema. J. Exper. Med., 1917, 26, 201.

Loeb, Leo, The mechanism in the development of pulmonary edema. Proc. Soc. Exper. Biol. and Med., 1928, 25, 321.
Johnson, S., Experimental production and prevention of acute edema of the lungs in rabbits. Proc. Soc. Exper. Biol. and Med., 1927, 25, 181.

Plesch. Quoted as a personal communication by Campbell, A., and Poulton, E. P., Oxygen and Carbon Dioxide Therapy. Oxford University Press, London, 1934.

Poulton, E. P., Personal communication.

11. Harrison, T. R., Failure of the Circulation. Williams and Wilkins Co., Baltimore, 1935, p. 129.

12. Barach, A. L., and Richards, D. W., Jr., Effects of treatment with oxygen in cardiac failure. Arch. Int. Med., 1931, 48, 325.

Barach, A. L., The treatment of asphyxia in clinical disease with especial reference to recent developments in the use of oxygen in heart disease. N. Y. State J. Med., 1934, 34, 672.

Richards, D. W., and Barach, A. L., Prolonged residence in high oxygen atmospheres. Effects on normal individuals and on patients with chronic cardiac and pulmonary insufficiency. Quart. J. Med., 1934, 3, 437.

Poulton, E. P., Dyspnea. Guy's Hosp. Gaz., 1935, 49, 346. 